\title{
Order and duration of stimuli are important determinants of reactivation
}

\author{
H. MOORE ARNOLD and NORMAN E. SPEAR \\ Binghamton University, Binghamton, New York
}

\begin{abstract}
Prior cuing treatments intended to alleviate the forgetting of a conditioned aversion to an odor were tested with 18-day-old rats. Previous experiments had shown that when such pups were conditioned with the use of a CS-/CS + procedure, pretest presentation of the CS- or US, but not the CS+, alleviated the forgetting otherwise seen after a 3-h retention interval. In Experiment 1 , it was determined that the forgetting was not alleviated if the CS- was either preceded or followed by presentation of the CS + , despite the fact that the CS-/CS+ ordering mimicked that of original conditioning. Experiment 2 was an examination of the balance of extinction and reactivation effects caused by presenting the $\mathrm{CS}+$ for varying durations following the 3-h retention interval. The forgetting over this interval was alleviated if the CS+ was presented for 5 or $15 \mathrm{sec}$, but not $30 \mathrm{sec}$. With an increase in duration of exposure from 15 to $30 \mathrm{sec}$, the consequences of the CS+ as a prior cuing treatment apparently shifted from reactivation to extinction. Experiment 3 was a test of the interaction between the consequences of different lengths of CS+ exposure and the effectiveness of adding $\mathrm{CS}-$ to the $\mathrm{CS}+$ as a reactivation treatment. The varied effectiveness of reactivation treatments is discussed in terms of a change in stimulus conditions from training to reactivation.
\end{abstract}

The necessary and sufficient conditions for the alleviation of forgetting remain unsettled in memory research. One effective treatment in the alleviation of forgetting is the presentation of a reminder, or prior cue. Many authors have noted the importance of such procedures, which are typically referred to as reactivation treatments (Gordon, 1981, 1983; Rovee-Collier, Sullivan, Enright, Lucas, \& Fagen, 1980; Spear, 1978; Spear, Miller, \& Jagielo, 1990; Spear \& Parsons, 1976). A reactivation treatment involves exposing the subject to a specified aspect of the original training episode prior to a retention test. Reactivation treatments have been used to alleviate forgetting that results from many different sources, such as long retention intervals (Gordon, Smith, \& Katz, 1979; Spear \& Parsons, 1976), short retention intervals (Feldman \& Gordon, 1979; Gordon \& Feldman, 1978), and electroconvulsive shock (R. R. Miller \& Springer, 1973). Reactivation treatments have also been shown to alleviate forgetting in preweanling animals (Campbell \& Jaynes, 1966) and in human infants (Rovee-Collier et al., 1980).

In a recent series of experiments, J. S. Miller, Jagielo, and Spear $(1989,1990,1991)$ investigated reactivation in preweanling rat pups. J. S. Miller et al. (1989) used a one-trial conditioning procedure in which preweanling

This research was supported in part by a grant from the National Institute of Mental Health (1 R01 MH35219) to N.E.S. The authors would like to thank Ralph R. Miller, Stanley R. Scobie, and David L. McKinzie for their comments on an early version of the manuscript. We would also like to thank Teri Tanenhaus for secretarial assistance in preparing the manuscript, Norman Richter for technical assistance, and Heather Rabine for assistance in collecting the data. Requests for reprints may be addressed to $\mathbf{N}$. E. Spear, Center for Developmental Psychobiology, Binghamtom University, Binghamton, NY 13902. rats were exposed to a lemon odor ( $\mathrm{CS}-$ ) prior to the pairing of a second odor, methyl salicylate (CS+), with footshock, to condition an aversion to the CS + . The conditioned aversion was evident up to $2 \mathrm{~h}$ after training, but not $3 \mathrm{~h}$ after training. J. S. Miller et al. $(1990,1991)$ then used this procedure to investigate the differential effectiveness of various reactivation treatments on the expression of the learned but forgotten aversion to the CS+. J. S. Miller et al. (1990) observed that the US alone and the CS - were both effective retrieval cues; responding to the $\mathrm{CS}+$ was restored in subjects that received either of these reactivation treatments. Interestingly, responding was not restored in rats given the CS+ as the reactivation cue.

It is not immediately obvious why exposure to the CSshould be a more effective retrieval cue than exposure to the CS + . If a reactivation treatment serves to "remind" the animals about the CS + , one would expect that the $\mathrm{CS}+$ itself should be the most effective reactivation cue. However, Campbell and Randall (1976) observed that after acquisition of a discrimination between the $S+$ and $\mathrm{S}-$ in terms of appetitive leverpressing, multiple presentations of the $S$ - during the retention interval tended to be more effective as a reinstatement procedure than presenting the $S+$. (The $S+$ was reinforced during the reinstatement phase.) This difference in retention occurred entirely in terms of response to the $S-$, however, with response to the $S+$ about equal for the two groups. Moreover, their procedures for instrumental appetitive conditioning were obviously much different from those reported by J. S. Miller et al. (1991), who did Pavlovian aversive conditioning. In addition, this reinstatement paradigm of Campbell and Randall involved numerous distributed pre- 
sentations of the cues after original training and thus may or may not be relevant to studies with a single presentation of the reminder cue. In one such study, retention of appetitive instrumental conditioning was also tested, with human infants; the $S+$ rather than the $S$ - was found to be a more effective reactivation treatment (Fagan, Yengo, Rovee-Collier, \& Enright, 1981).

One explanation for the poor performance of the CS + reactivation treatment, offered by $J$. S. Miller et al. (1990), is that in training the CS - precedes or predicts the CS+/US pairing, potentially acting as a form of occasion setter (Holland, 1983, 1986). Thus, when the CS+ is presented alone, forgetting is not alleviated, because the stimulus complex of the reactivation treatment differs significantly from that of conditioning, in which the rats learn that the CS + is aversive if preceded by the CS - . As pointed out by J. S. Miller et al. (1989), the training procedures typically used to produce an effective occasion setter are quite different from those used by J. S. Miller et al. $(1989,1990)$ in their procedures. An occasion setter is thought to disambiguate the relationship between a CS and a US by signaling when the CS will be followed by reinforcement (Holland, 1983). Training typically involves the presentation of a stimulus prior to the pairing of another stimulus with reinforcement, as well as separate trials in which the CS terminates in nonreinforcement $(A / B+, B-)$. The acquisition of this discrimination involves numerous trials before the animals are able to learn the contingency, so the procedure by $\mathbf{J}$. S. Miller et al. $(1990,1991)$ is not strictly analogous to that used in occasion-setting procedures. Despite the differences however, evidence suggests that the CS- is an effective reactivation cue, in part because of its ability to signal the CS +/US pairing (J. S. Miller, Jagielo, \& Spear, 1992).

In these experiments, J. S. Miller et al. (1992) demonstrated that the CS - was a more effective reactivation treatment if it had preceded the CS + during training than if it had followed the CS + . These results were not confounded by differences in learning that might have been expected since the latter treatment ( $\mathrm{CS}+/ \mathrm{CS}-$ ) is more typical of a discriminative training paradigm. Apparently there are no differences in conditioning in 18-day-old pups trained with the CS- preceding or following the CS+.

Another reason why the CS + might be less effective than the $\mathrm{CS}$ - as a reactivation cue is that the $\mathrm{CS}+$ odor in the absence of footshock (reactivation treatment) might not be perceived as the same CS + experienced in the presence of footshock during original conditioning. This change in properties of the CS + between training and cuing may have been great enough to render the CS + ineffective as a retrieval cue. This stimulus-change hypothesis has been suggested previously (Hamberg \& Spear, 1978 ) in relation to a discrimination task, to account for the failure of certain aspects of the training situation to alleviate forgetting.

In the present series of experiments, we used the procedure developed by J. S. Miller et al. (1991), in order to determine further the factors that govern whether a specific stimulus will reactivate an apparently forgotten memory.

\section{EXPERIMENT IA}

If the $\mathrm{CS}+$ has not been an effective reactivation treatment because of the absence of the US, the CS - , or the same sequence of these events used for conditioning - as the stimulus change view would suggest--then, presenting these events with the CS + may make it an effective reactivation treatment. The first experiment was an attempt to find conditions in which the CS + would alleviate forgetting, as well as to replicate the ineffectiveness of the CS + , relative to the CS - and US, observed in previous studies (J. S. Miller et al., 1991).

Animals were trained and then cued with one of several different reactivation treatments. Four of these conditions (no cue, CS + alone, US alone, and CS - alone) served to replicate findings by J. S. Miller et al. (1991). The remaining conditions were designed to assess whether stimulus change between training and the reactivation treatment determines the effectiveness of reactivation treatments; in these conditions, stimuli were presented either as they occurred during training (CS $+/ \mathrm{US}$, $\mathrm{CS}-/ \mathrm{CS}+$ ) or in a manner that was different from how they occurred during training $(\mathrm{CS}+/ \mathrm{CS}-)$.

\section{Method}

Subjects. The subjects were 134 naive 18-day-old SpragueDawley-derived male and female rat pups from our breeding colony at Binghamton University. These subjects were housed with their parents and siblings until participation in the experiment. In this and in all subsequent experiments, there was never more than 1 pup from a litter in any given group. All subjects were maintained in a temperature-controlled colony room with a 16:8-h light:dark cycle, with light onset at $0700 \mathrm{~h}$. The research was conducted during the light portion of the cycle.

Apparatus. All experimental treatments and testing took place in one of three different rooms. Each room was similar in size $(8 \times 8 \times 7.5 \mathrm{ft})$, with shielded fluorescent lighting along one wall. Conditioning took place in a clear Plexiglas chamber $(10.16 \times$ $15.24 \times 20.32 \mathrm{~cm}$ ) with a stainless steel grid floor. The floor consisted of $2-\mathrm{mm}$ rods that were positioned $4 \mathrm{~mm}$ apart. Odorants were used as stimuli, placed on cotton underneath the grid floor of the conditioning chamber. The CS - was $1.0 \mathrm{ml}$ of imitation almond extract (McCormick). This was presented in a room different from the room used for $\mathrm{CS}+$ conditioning, but in an identical chamber. The CS + was $2.0 \mathrm{ml}$ of methyl salicylate (Aldrich Chemical Co.). The time required to transport subjects from the $\mathrm{CS}$ - room to the CS + room was approximately $15 \mathrm{sec}$. Preference testing took place in a third room $20 \mathrm{ft}$ from the rooms where the $\mathrm{CS}-$ and $\mathrm{CS}+$ had been presented. During the retention intervals and prior to the onset of conditioning, the subjects were isolated in a holding cage $(13 \times 11 \times 15 \mathrm{~cm})$ lined with clean shavings located in the hallway between the CS + room and the testing room.

Testing was conducted in a clear Plexiglas chamber $(27.94 \times$ $10.64 \times 12.38 \mathrm{~cm}$ ) with a layer of clean shavings on the chamber floor. The CS + was spread on cotton and inserted into an opening located $13 \mathrm{~mm}$ above the floor at one end of the preference chamber. An alternative odor ( $1.0 \mathrm{ml}$ of lemon oil, Humco Inc.) was spread on cotton and inserted into an identical opening at the opposite end of the chamber. The concentrations of the odorants were based on findings from pilot experiments, in which animals that received the odors and footshock explicitly unpaired preferred 
methyl salicylate to lemon on the preference test. Footshock was delivered by a Grason-Stadler shock generator, Model L5489.

Design and Procedure. Each treatment condition included 7-9 animals, with no more than 1 subject taken from a single litter. Prior to any experimental manipulation, all subjects were placed in the holding cage.

For conditioning, each subject was placed for $30 \mathrm{sec}$ into the compartment containing the CS - odor (almond). Immediately following this exposure, the subjects were moved to a different room and placed for $30 \mathrm{sec}$ into the compartment containing the CS+ odor (methyl salicylate). During the $30 \mathrm{sec}$ in the CS + chamber, the subjects in the paired conditions received three 1.6-mA scrambled footshocks of 3-sec duration, during Seconds 8-10, 18-20, and 28-30. The subjects in the unpaired control conditions (one for each treatment condition) received three identical footshocks during a 30-sec placement into a nonodorized clear Plexiglas chamber $20 \mathrm{~min}$ prior to presentation of the CS - and CS + odors (this was the same chamber that was used to present the CS + prior to any odor's being introduced into the room).

Following the conditioning treatments, all subjects remained in the holding cage until the end of a 3-h retention interval, at which time they were given a $60-\mathrm{sec}$ test of their preference between the locations of the CS + odor and a novel odor (lemon). Five minutes prior to this test, the subjects in the prior cuing conditions were removed from the holding cage, exposed to a selected element of the conditioning episode, and returned to the holding cage. These elements were the following: the US used during training (30 sec in the nonodorized $\mathrm{CS}+$ chamber with the US occurring throughout Seconds 8-10, 18-20, and 28-30; Groups US Alone); a 30-sec exposure to the methyl odor (Groups CS + Alone); a 30-sec exposure to the $\mathrm{CS}+$, during which time three 3 -sec shocks were given during Seconds 8-10, 18-20, and 27-30 (Groups CS+/US); a 30-sec exposure to the $\mathrm{CS}+$ followed by a $30-\mathrm{sec}$ exposure to the $\mathrm{CS}-$ (Groups $\mathrm{CS}+/ \mathrm{CS}-$ ); $30 \mathrm{sec}$ of exposure to the $\mathrm{CS}-$ followed immediately by $30 \mathrm{sec}$ of exposure to the $\mathrm{CS}+($ Groups $\mathrm{CS}-/ \mathrm{CS}+$ ); and a 30-sec exposure to the CS - alone (Groups CS - Alone). All these reactivation treatments occurred in the same chambers that were used during conditioning. There was also one condition in which no prior cue was given (Groups No Cue); these subjects remained in the holding cage for the entire retention interval prior to the test.

The animals were placed in the center of the test chamber facing a sidewall, so that they were not facing either odor. The direction of the snout was alternated between animals so that $50 \%$ of the animals were started in each direction. The time spent on the methyl side of the chamber was recorded during a 60 -sec test. Criterion for a preference was defined as the animal's crossing a midline with both ears. If only one ear crossed, the animal was scored on the side with the majority of the body.

\section{Results and Discussion}

The results of the preference test are presented in Figure 1. A 7 (treatment condition) $\times 2$ (pairing; paired vs. unpaired) analysis of variance (ANOVA) revealed a significant effect of treatment $[F(6,115)=2.51, p<.03]$ and an effect of pairing $[F(1,115)=27.75, p<.01]$. In addition, the interaction between treatment condition and pairing was significant $[F(6,115)=3.37, p<.01]$.

Pairwise comparisons were conducted with the Fisher test (Keppel, 1982); the significance level was set at $p<$ .05. Paired groups differed significantly from their respective unpaired controls in three conditions: US alone, $\mathrm{CS}+/ \mathrm{US}$, and CS - alone. Paired groups in the no-cue,

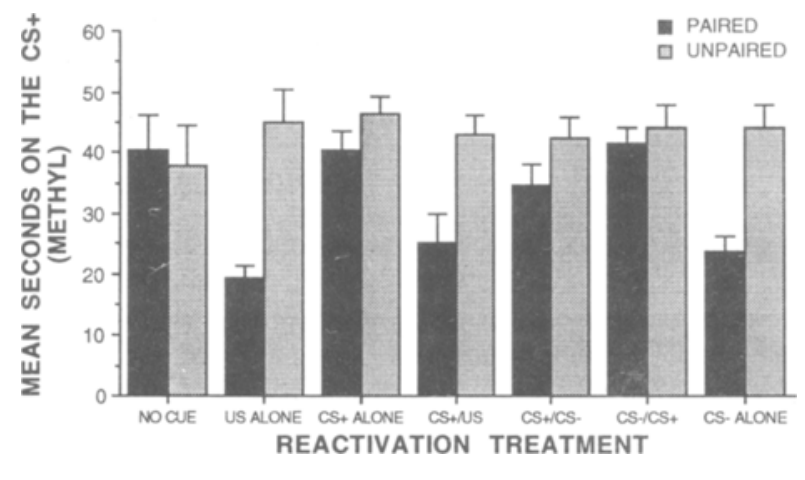

Figure 1. Mean seconds spent on the CS + (methyl odor) side of the chamber during the odor preference test by subjects given various reactivation treatments, except no cue, which received no reactivation treatment. (Vertical lines indicate standard errors of the mean.)

$\mathrm{CS}+$ alone, $\mathrm{CS}+/ \mathrm{CS}-$, and $\mathrm{CS}-/ \mathrm{CS}+$ conditions did not differ from their unpaired controls. Finally, no unpaired group differed from any other unpaired group (all $p s>.05$ ).

The pattern of results in this experiment replicates systematically that found by J. S. Miller et al. $(1990,1991)$, who used a slightly different set of parameters. The most notable difference was that, in the present experiment, the odors that served as the CS - and the novel test odor were different from those used by J. S. Miller et al. in their studies. Animals that received no reactivation treatment or the $\mathrm{CS}+$ as the treatment failed to express an aversion to the CS +. However, animals given the CSor the US as a reactivation treatment did express an aversion to the $\mathrm{CS}+$.

If the CS + merely fails to reactivate the memory and has no other effect as a reactivation treatment, we expected that the CS - would continue to alleviate forgetting when presented with the $\mathrm{CS}+$ as a reactivation treatment, regardless of the order in which these two stimuli were presented. However, if instead the rat had learned during conditioning that the $\mathrm{CS}-$ predicts the occurrence of the $\mathrm{CS}+$, we expected that the CS-/CS + condition would be a more effective cue than the $\mathrm{CS}+/ \mathrm{CS}-$ condition. What was observed, however, was that neither condition was an effective reactivation treatment. This suggests that the CS + inhibited the CS - from cuing the memory, or, perhaps, extinguished the aversion during the $30 \mathrm{sec}$ of its exposure.

\section{EXPERIMENT 1B}

This experiment was conducted simultaneously with the previous experiment. We wanted to ensure that the effects were not specific to the odors used as the CS - and CS +, almond and methyl salicylate, respectively. To further extend the generalizability of the reactivation phenomena, 
four treatment conditions from Experiment 1A were run with the odors of the CS - and CS + reversed.

\section{Method}

Subjects. The subjects were forty 18 -day-old pups from five different litters. The rearing conditions were as those described in Experiment $1 \mathrm{~A}$.

Apparatus and Procedure. The apparatus was exactly as in Experiment 1. The procedure was also the same as in Experiment 1 , except for the following changes. The $\mathrm{CS}+$ was $1.0 \mathrm{ml}$ of almond odor and the CS - was $2.0 \mathrm{ml}$ of methyl salicylate. The novel odor for the preference test remained $1.0 \mathrm{ml}$ of lemon oil. Only three reactivation conditions were used: the US used during training (30 $\mathrm{sec}$ in an unodorized chamber with the US occurring throughout Seconds 8-10, 18-20, and 28-30; Groups US Only); a 30-sec exposure to the methyl salicylate odor (Groups CS - Only); and a 30-sec exposure to the almond odor (Groups CS + Only). There was also one condition in which no prior cue was given (Groups No Cue). Each condition included paired and unpaired groups, as in Experiment 1 .

\section{Results and Discussion}

The means for the odor preference test are presented in Figure 2. A 4 (reactivation treatment) $\times 2$ (pairing: paired vs. unpaired) ANOVA revealed a significant main effect of treatment $[F(3,32)=11.49, p<.01]$ and of pairing $[F(1,32)=59.25, p<.01]$. In addition, the interaction was significant $[F(3,32)=5.29, p<.01]$. Pairwise comparisons (Fisher test) confirmed that paired groups in the no-cue, US only, and CS - only conditions differed from their unpaired controls. The CS + only paired condition did not differ from its unpaired control, and these subjects spent significantly more time on the $\mathrm{CS}+$ side of the test chamber than did those in the nocue paired condition. In addition, the no-cue paired group spent significantly more time on the CS + than did the US only and CS - only paired groups.

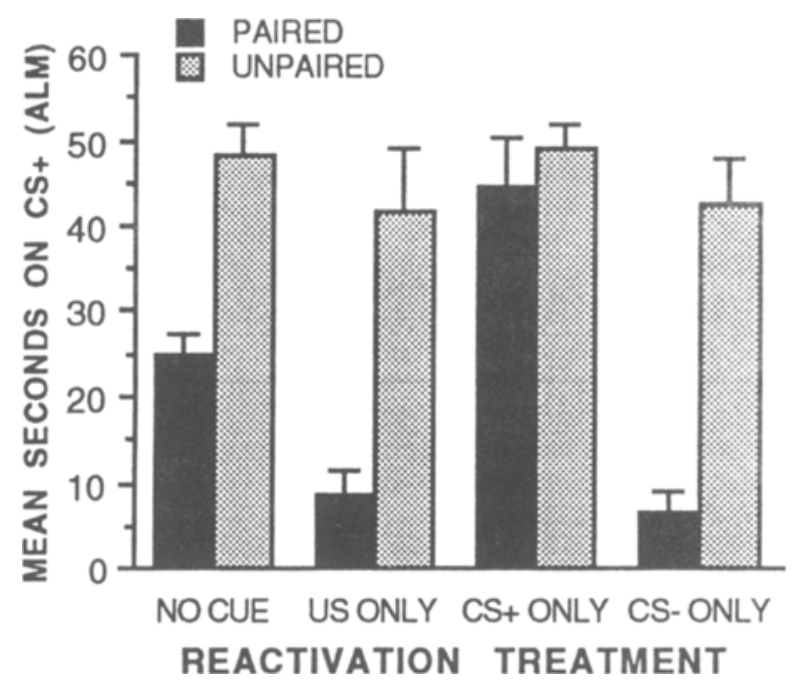

Figure 2. Mean seconds spent on the CS + (almond odor) side of the chamber during the odor preference test by subjects given cuing treatments with the CS + (CS + only), CS - (CS - only), or footshock (US only). No cue indicates that no reactivation treatment was given. (Vertical lines indicate standard errors of the mean.)
These results support the findings from Experiment $1 \mathrm{~A}$ and those of Miller et al. (1991). Of primary note is that the CS + only paired animals did not differ from their unpaired controls and that the US only and CS- only groups spent less time on the CS + than did the no-cue animals, suggesting that the reactivation treatments were effective. The only anomalous result was that the no-cue paired animals spent less time on the $\mathrm{CS}+$ than did their unpaired controls, indicating that the paired animals could still express, to a limited degree, the memory of original training. These data serve to extend the generality of the effects that were seen in Experiment 1A.

It is interesting that the CS + not only failed to cue the animals as we expected, but actually decreased the aversion in the CS + only group in comparison with the nocue group. These results, taken together with those of Experiment $1 \mathrm{~A}$, led us to examine the possibility that the conditioned aversion to the $\mathrm{CS}+$ was undergoing extinction during the brief exposure to the $\mathrm{CS}+$ that constituted the reactivation procedure.

\section{EXPERIMENT 2}

J. S. Miller et al. (1990) tested the possibility that extinction of the aversion to the CS + was responsible for its failure to alleviate forgetting by exposing the animals for $30 \mathrm{sec}$ to the CS + immediately after conditioning. An immediate test indicated no loss of responding to the CS + . Experiment 2 was a test of the possibility that $30 \mathrm{sec}$ in the presence of $\mathrm{CS}+$ might nevertheless constitute an effective extinction procedure if given after a significant delay following conditioning. The animals were trained as in Experiment $1 \mathrm{~A}$, and then, after a 3-h retention interval, they were given no cue, the CS - for $30 \mathrm{sec}$, or the $\mathrm{CS}+$ for 5,15 , or $30 \mathrm{sec}$. If the aversion to the CS + is extinguished during the 30 -sec exposure, shorter exposure to the CS + might serve to cue the memory without extinguishing it (Gordon et al., 1979).

\section{Method}

Subjects. The subjects were ninety 18-day-old rat pups similar to those described in Experiment $1 \mathrm{~A}$.

Apparatus and Procedure. The apparatus was identical to that used in Experiment 1A. The procedure was also the same as that of Experiment 1A, except for the specific reactivation treatments given at the end of the retention interval. In Experiment 2, subjects received one of the following: a 5-, 15-, or 30-sec exposure to the $\mathrm{CS}+$ only (Groups $\mathrm{CS}+5, \mathrm{CS}+15$, and $\mathrm{CS}+30$ ); a 30sec exposure to the CS- (Group CS- 30); or no reactivation treatment (Group No Cue). The animals were then tested as described previously.

\section{Results and Discussion}

Figure 3 shows the means of the preference test. A 5 (treatment conditions) $\times 2$ (pairing: paired vs. unpaired) ANOVA revealed a significant effect of treatment $[F(4,80)=3.32, p<.05]$, a significant effect of pairing $[F(1,80)=20.15, p<.01]$, and a significant interaction between treatment and pairing $[F(4,80)=2.46$, $p<.05]$. 


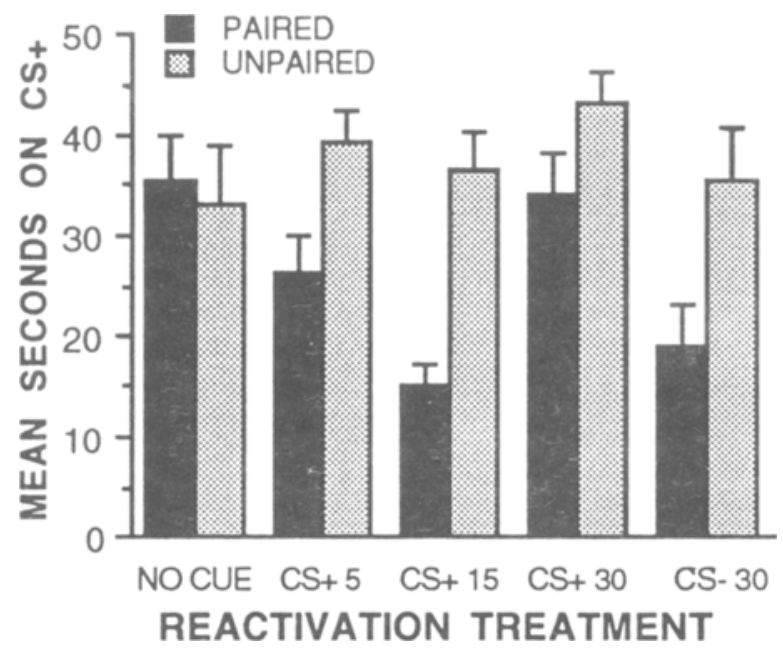

Figure 3. Mean seconds spent on the CS + (methyl odor) side of the chamber during the odor preference test by subjects given no reactivation treatment (no cue), 5,15 , or $30 \mathrm{sec}$ of exposure to the $\mathrm{CS}+(\mathrm{CS}+5, \mathrm{CS}+15$, and $\mathrm{CS}+30)$ or $30 \mathrm{sec}$ of exposure to the CS- (CS- 30). (Vertical lines indicate standard errors of the mean.)

Pairwise comparisons (Fisher test) revealed that the nocue and the CS +30 paired groups did not differ from their unpaired controls, whereas the CS $+5, \mathrm{CS}+15$, and the CS -30 paired groups did spend significantly less time on the CS + side of the chamber than did their respective unpaired controls. In addition, Groups CS +5 , $\mathrm{CS}+15$, and CS -30 all spent less time on the CS + side of the preference chamber than did Groups No Cue and $\mathrm{CS}+30$. No cue and $\mathrm{CS}+30 \mathrm{did}$ not differ from each other.

These results are consistent with the idea that the apparent failure of the $\mathrm{CS}+$ as a reactivation treatment in prior studies was due to extinction of the aversion to the CS + resulting from the full $30-\mathrm{sec}$ exposure to it during the reactivation treatment. This agrees with findings reported by Gordon et al. (1979), who used the CS + in an active avoidance procedure as a reactivation cue. Using adult animals, Gordon et al. found that $15 \mathrm{sec}$ of exposure to the CS after an extended interval served to restore responding, but $75 \mathrm{sec}$ of exposure to the CS did not. They suggested that the initial time spent in the presence of the CS + serves to cue the memory, whereas additional time spent in the presence of the CS + after the memory is retrieved can result in extinction. In other words, after the memory is retrieved, the animals begin learning the new contingency.

\section{EXPERIMENT 3}

The last experiment was designed to return to our concern for the stimulus characteristics that determine the effectiveness of a reactivation procedure. In Experiment $1 \mathrm{~A}$ we assessed whether stimulus change contributed to the failure of the CS + to alleviate forgetting when the $\mathrm{CS}+$ was presented for the same duration as in condi- tioning. Experiment 2 showed that $\mathrm{CS}+$ is, however, capable of alleviating forgetting in this procedure if its presentation is sufficiently brief. Experiment 3 was designed to assess whether the order of stimulus presentation during the reactivation treatment, which could potentially include a significant change from the conditioning stimuli (in terms of sequencing), also served to prevent the alleviation of forgetting when the $\mathrm{CS}+$ was presented in conjunction with the $\mathrm{CS}-(\mathrm{CS}-/ \mathrm{CS}+$ and $\mathrm{CS}+/ \mathrm{CS}-$, in Experiment 1A).

In Experiment 3, we also sought to assess the hypothesis proposed by Gordon et al. (1979; Gordon, 1981)that presentation of the CS + has different effects, depending on whether the training memory is active or not at the time of $\mathrm{CS}+$ presentation. If the training memory was made active prior to the presentation of the $\mathrm{CS}+$, we expected that a shorter duration of the CS + would be sufficient for acquisition of the extinction contingency. However, if the training memory was not active during initial exposure to the $\mathrm{CS}+$, then a brief $\mathrm{CS}+$ exposure might be insufficient for extinction to occur.

\section{Method}

Subjects. The subjects were seventy-two 18-day-old rat pups similar to those described in Experiment $1 \mathrm{~A}$.

Apparatus and Procedure. The apparatus was identical to that used in Experiment 1A. The procedure was also the same as that in Experiment 1A, except for the specific reactivation treatments given at the end of the retention interval and the absence of unpaired groups. Given the consistency of the unpaired conditions in Experiments $1 \mathrm{~A}, 1 \mathrm{~B}$, and 2, as well as in several prior experiments by J. S. Miller et al. $(1989,1990,1991)$, we felt justified in omitting these groups from Experiment 3. Also, the no-cue animals in all the experiments mentioned above (except Experiment 1B, in which different stimuli were used) did not differ from those in the unpaired conditions. The no-cue condition in Experiment 3 therefore allowed comparisons with a condition in which subjects were responding at a level indicative of no learning. In this experiment, the subjects received one of the following: no reactivation treatment (Group No Cue); a 5-sec or 15-sec exposure to the CS + only (Groups CS +5 and $\mathrm{CS}+15$ ); a 30 -sec exposure to the CS(Group CS - 30); the CS - for $30 \mathrm{sec}$ followed immediately by the $\mathrm{CS}+$ for 5 or $15 \mathrm{sec}$ (Groups CS - 30/CS +5 and CS - 30/CS + 15 ); or the $\mathrm{CS}+$ for 5 or $15 \mathrm{sec}$ followed immediately by the CS(Groups CS $+5 / \mathrm{CS}-30$ and $\mathrm{CS}+15 / \mathrm{CS}-30$ ). The animals were then tested as described previously.

\section{Results and Discussion}

The means for Experiment 3 are presented in Figure 4. A one-way ANOVA revealed a significant effect of reactivation treatment $[F(6,74)=6.62, p<.001]$. Pairwise comparisons indicated that Groups CS $-30, \mathrm{CS}+5$, $\mathrm{CS}+15$, and $\mathrm{CS}+15 / \mathrm{CS}-30$ all differed significantly from the no-cue group. Groups $\mathrm{CS}-30 / \mathrm{CS}+5, \mathrm{CS}-$ $30 / \mathrm{CS}+15$, and CS $+5 / \mathrm{CS}-30$ did not differ from each other or the no-cue group. This indicates that these conditions $(\mathrm{CS}-30 / \mathrm{CS}+5, \mathrm{CS}-30 / \mathrm{CS}+15$, and $\mathrm{CS}+$ $5 / \mathrm{CS}-30$ ) did not alleviate the forgetting incurred over the 3-h retention interval.

These data replicated the findings of Experiment 2 in showing that the CS + is an effective treatment for alleviating forgetting in the present circumstances, if given for 


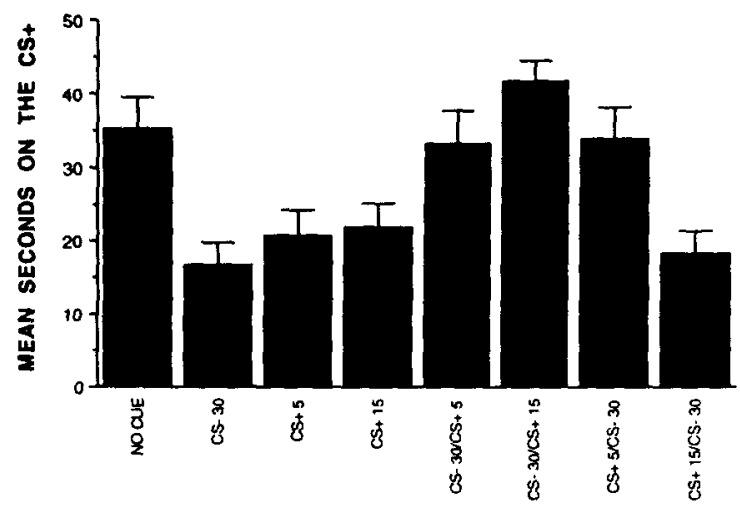

REACTIVATION TREATMENT

Figure 4. Mean seconds spent on the CS + (methyl odor) side of the chamber during the odor preference test by subjects given various prior cuing treatments. In the no-cue condition, no prior cue was given. Numbers after $\mathrm{CS}+$ and $\mathrm{CS}-$ indicate the duration (in seconds) of exposure to the stimuli during the reactivation treatment only. (Vertical lines indicate standard errors of the mean.)

a short enough duration so as not to allow extinction. This is evidenced by performance of the CS +5 and $C S+15$ groups relative to that of the $\mathrm{CS}+30$ group.

It is striking in these data that both groups given the CS- prior to the CS+ (Groups CS-30/CS + 5 and $\mathrm{CS}-30 / \mathrm{CS}+15$ ) failed to express an aversion to the $\mathrm{CS}+$ on the preference test. This suggests that as little as $5 \mathrm{sec}$ of exposure to the CS + can extinguish the aversion if the forgotten memory is made active by exposure to the CS - just prior to exposure to the CS +. In this experiment, $30 \mathrm{sec}$ of exposure to the CS - was sufficient to reactivate the memory, as can be seen by the performance of Group CS - 30. Exposure to the CS + immediately following the CS - , however, disrupted the consequence of this reactivation, presumably by allowing the animals enough time to learn the extinction contingency despite the short duration of the stimuli. However, when the CS + preceded the CS - in Group CS + 15/CS - 30, the CS + was the cue that served to activate the memory. The $30 \mathrm{sec}$ of exposure to the CS - apparently did not serve to extinguish the aversion to methyl odor, suggesting that the animals were not able to learn the extinction contingency in the absence of the CS +. In fact, there is little reason to suspect that the CS - could serve to extinguish the memory other than by generalization from the CSto the CS+. The performance of Group CS $+5 / \mathrm{CS}-30$ does not appear to support this interpretation, however. This group's anomalous results are discussed below in more detail.

These findings generally support the hypothesis of Gordon et al. (1979; Gordon, 1981)-that at the time of reactivation, exposure to the CS+ will have different effects, depending on whether the training memory is active or not prior to $\mathrm{CS}+$ exposure.

\section{GENERAL DISCUSSION}

The present series of experiments replicates and extends previous findings concerning the alleviation of forgetting in preweanling rat pups (J. S. Miller et al., 1989, 1990, 1991). One purpose of this series was to determine why the CS + had failed in several earlier experiments to reactivate the memory of the aversion, despite excellent retention after reactivation treatments that included other elements of the conditioning procedure (e.g., the CS-). Experiments 1A, 1B, and 2 seem to have answered this question, yet differently than we had expected. The CS+ does, in fact, reactivate the memory when the exposure is sufficiently brief $(5-15 \mathrm{sec})$ so as not to induce new learning of the contingency between the CS + and nonreinforcement.

These findings, taken together with those of J. S. Miller et al. (1990), which indicated no extinction of the CS + after $30 \mathrm{sec}$ of exposure when the exposure and test occurred immediately after testing, suggest an interaction between the duration of CS + exposure and the length of the retention interval. Unfortunately, few data speak to this issue, and most have been derived from tests involving many conditioning trials and appetitive reinforcers. In such studies, researchers observed an increase in resistance to extinction over longer retention intervals (e.g., Aiken \& Gibson, 1965; Hilgard \& Campbell, 1936; Spear \& Spitzner, 1967; Youtz, 1938). This effect has been explained as a result of the generalization gradient's flattening as the retention interval increases, thus weakening the discrimination between acquisition and extinction. What implications these studies have for the present, brief aversive conditioning procedure in preweanling rats is not clear. The present series of experiments does not demonstrate an increased resistance to extinction over time. On the contrary, the present data suggest that the extinction of the CS + is more rapid after a delay than immediately after conditioning (cf. J. S. Miller et al., 1990). However, it may be the case that what has appeared as resistance to extinction in previous experiments may have been the result of the initial extinction trials' serving to reactivate the training memory, and that learning of the new contingency (extinction) did not begin until reactivation was complete.

The finding that the CS + is indeed an effective reactivation treatment contrasts with the idea, expressed by J. S. Miller et al. (1991) and in the present report, that the CS + may be ineffective as a reactivation treatment because of the changing nature of the stimulus properties of the CS+ from training to cuing. Evidence against the stimuluschange hypothesis can be observed by consideration of Group CS + 15/CS - 30 from Experiment 3. Despite a change in CS + duration as well as in stimulus order, this treatment was effective in alleviating forgetting. It is possible that the relative ineffectiveness of the CS $+5 / C S-$ 30 condition in Experiment 3 (in which the CS + was pre- 
sented for only $5 \mathrm{sec}$ prior to the CS-) may in fact be attributed to stimulus change from conditioning. This treatment differed more from original training than did $\mathrm{CS}+15 / \mathrm{CS}-30$. Until further data are collected, however, this possibility should be viewed with caution. We conclude from these data that although stimulus change may remain a reasonable explanation for the failure of certain reactivation treatments (Hamberg \& Spear, 1978), it is not the primary determinant of the effectiveness of reactivation treatments tested in this series and in related studies (J. S. Miller et al., 1989, 1990, 1991).

In a similar test on the effects of the duration of the $\mathrm{CS}+$ as a reactivation cue, using adult animals, Gordon et al. (1979) found that a brief exposure $(15 \mathrm{sec})$ reactivated a memory of active avoidance, whereas a longer exposure $(75 \mathrm{sec})$ extinguished the response. Gordon et al. suggested that when subjects were initially exposed to the $\mathrm{CS}+$, reactivation of the memory began. After the reactivation of the memory was complete, the organism was able to learn the new contingency involving the CS +, which in this case was that of nonreinforcement. In Experiment 3, we tested the implication that nonreinforced exposure to the CS + would affect performance differently, depending on whether the CS + was presented when the training memory was active. If the memory were active when the CS + was presented, the animal should incorporate new (extinction) contingencies into the memory immediately upon presentation of the $\mathrm{CS}+$. If the memory were not in an active state when the CS + was presented, the CS + should first serve to reactivate the memory before new information could be incorporated into the memory. Apparently, as little as $5 \mathrm{sec}$ in the presence of a nonreinforced CS + was sufficient for the animals to learn the extinction contingency, provided that memory had been made active by presentation of the CS - .

Under this framework, presentation of the CS+ prior to the CS - should serve to reactivate the memory, yet not extinguish it. Experiment 3 is ambiguous on this point. Cuing with the CS+ for $15 \mathrm{sec}$ prior to the presentation of the CS - was an effective reactivation treatment, as predicted. However, the absence of a similar cuing effect when the CS + occurred for only $5 \mathrm{sec}$ prior to the CS makes the interpretation difficult. One could argue that there was some generalized extinction of the CS + by presentation of the CS-, but if this were the case, the same pattern of results would be expected for the group given the $\mathrm{CS}+$ for 5 or $15 \mathrm{sec}$ prior to the CS - Explanation of the results in terms of the total duration of exposure to the cues (both CS + and CS - ) is similarly negated by the performance of rats given the CS $+15 / \mathrm{CS}-30$ prior cuing condition.

Perhaps after presentation of a reactivation cue, significant time is needed before the memory can become active (Fagan \& Rovee-Collier, 1983). The CS + 5/CS - 30 group was moved from one odor to another more rapidly than any other. All other conditions had at least $10 \mathrm{sec}$ or longer before the presentation of a second odor. Although the CS +5 group was also moved out of the $\mathrm{CS}+$ after $5 \mathrm{sec}$, this group was returned to the holding cage, whereas the CS $+5 / \mathrm{CS}-30$ group was moved into the presence of the CS- (another odor). It is possible that the ineffective cuing with the CS $+5 / \mathrm{CS}-30$ treatment was due to a failure to adequately activate the memory prior to further manipulations by the experimenter. In other words, moving the animals into another odor before the memory is made active may somehow disrupt the reactivation process. Imposing a slight delay $(5-10 \mathrm{sec})$ between the $\mathrm{CS}+$ and $\mathrm{CS}-$ presentations during the reactivation treatment would allow one to assess this possibility.

Although stimulus change seems not to be the primary factor responsible for ineffective cuing with the $\mathrm{CS}+$, the extent to which stimulus change can play a role in determining effective reactivation treatments needs further examination. Understanding the necessary and sufficient conditions for reactivation and potential change (i.e., extinction) of a specific memory can be a valuable tool in determining the organization of a memory and the nature of the memory process.

\section{REFERENCES}

AIKEN, E. G., \& GiBson, K. L. (1965). Continuous and fixed ratio reinforcement effects in extinction one day and three weeks after acquisition. Psychonomic Science, 3, 527-528

Campbell, B. A., \& Jaynes, J. (1966). Reinstatement. Psychological Review, 73, 478-480.

Campbell, B. A., \& Randall, P. K. (1976). The effect of reinstatement stimulus conditions on the maintenance of long-term memory Developmental Psychobiology, 9, 325-333.

Fagan, J. W., \& Rovee-Collier, C. (1983). Memory retrieval: A timelocked process in infancy. Science, 222, 1349-1351.

Fagan, J. W., Yengo, L. A., Rovee-Collier, C. K., \& Enright, M. K. (1981). Reactivation of a visual discrimination in early infancy. Developmental Psychology, 17, 266-274.

FEldman, D. T., \& GORDON, W. C. (1979). The alleviation of shortterm retention decrements with reactivation. Learning \& Motivation, 10, 198-210.

GoRDON, W. C., (1981). Mechanisms of cue-induced retention enhancement. In N. E. Spear \& R. R. Miller (Eds.), Information processing in animals: Memory mechanisms (pp. 319-339). Hillsdale, NJ: Erlbaum.

GorDon, W. C. (1983). The malleability of memory in animals. In R. L. Mellgren (Ed.), Animal cognition and behavior (pp. 399-426). New York: Elsevier.

Gordon, W. C., \& FELdMAN, D. T. (1978). Reactivation induced interference in a short-term retention paradigm. Learning \& Motivation, 9, 164-178.

Gordon, W. C., Smith, G. J., \& Katz, D. S. (1979). Dual effects of response blocking following avoidance learning. Behavior Research \& Therapy, 17, 479-487.

Hamberg, J. M., \& Spear, N. E. (1978). Alleviation of forgetting of discrimination learning. Leaming \& Motivation, 9, 466-476.

Hilgard, E. R., \& Campbell, A. A. (1936). The course of acquisition and retention of conditioned eye-lid responses in man. Journal of Experimental Psychology, 19, 227-247.

Holland, P. C. (1983). Occasion-setting in Pavlovian feature positive discriminations. In M. L. Commons, R. J. Herrnstein, \& A. R. Wagner (Eds.), Quantitative analyses of behavior: Vol. 4. Discrimination processes (pp. 183-206). New York: Ballinger. 
Holland, P. C. (1986). Temporal determinants of occasion setting in feature-positive discriminations. Animal Learning \& Behavior, 14, $111-120$.

KePPEL, G. (1982). Design and analysis: A researcher's handbook. Englewood Cliffs, NJ: Prentice-Hall.

Miller, J. S., Jagielo, J. A., \& SPear, N. E. (1989). Age-related differences in short-term retention of separable elements of an odor aversion. Journal of Experimental Psychology: Animal Behavior Processes, 15, 194-201.

Miller, J. S., Jagielo, J. A., \& Spear, N. E. (1990). Alleviation of short-term forgetting: Effects of the $\mathrm{CS}$ - and other conditioning elements in prior cuing or as context during test. Learning \& Motivation, 21, 96-109.

Miller, J. S., Jagielo, J. A., \& Spear, N. E. (1991). Differential effectiveness of various prior cuing treatments on the reactivation and maintenance of memory. Journal of Experimental Psychology: Animal Behavior Processes, 17, 249-258.

Miller, J. S., Jagielo, J. A., \& Spear, N. E. (1992). The influence of the information value provided by prior-cuing treatment on the reactivation of memory in preweanling rats. Animal Learning \& Behavior, 20, 233-239.
Miller, R. R. \& Springer, A. D. (1973). Amnesia, consolidation and retrieval. Psychological Review, 80, 69-79.

Rovee-Colluer, C. K., Sullivan, M. W., Enrught, M., Lucas, D., \& FAGAN, J. W. (1980). Reactivation of infant memory. Science, 208, 1159-1161.

SPEAR, N. E. (1978). The processing of memories: Forgetting and retention. Hillsdale, NJ: Erlbaum.

Spear, N. E., Miller, J. S., \& Jagielo, J. A. (1990). Animal memory and learning. Annual Review of Psychology, 41, 169-211.

Spear, N. E., Parsons, P. J. (1976). Analysis of a reactivation treatment: Ontogenetic determinants of alleviated forgetting. In D. Medin, W. Roberts, \& R. Davis (Eds.), Processes in animal memory (pp. 135-166). Hillsdale, NJ: Erlbaum.

Spear, N. E., \& Spitzner, J. H. (1967). PRE in a T-maze brightness discrimination within and between Ss. Journal of Experimental Psychology, 73, 320-322.

Youtz, R. E. P. (1938). The change with time of a Thorndikian response in the rat. Journal of Experimental Psychology, 23, 128-140.

(Manuscript received October 14, 1992; revision accepted for publication February 28, 1993.) 